\title{
Herbert Spencer et la critique scientifique
}

Taine, Hennequin, Brunetière

\section{Marie Guthmüller}

\section{(2) OpenEdition}

Journals

Édition électronique

URL : http://journals.openedition.org/aes/286

DOI : 10.4000/aes.286

ISSN : 2258-093X

Éditeur

Laboratoire LISAA

Référence électronique

Marie Guthmüller, « Herbert Spencer et la critique scientifique », Arts et Savoirs [En ligne], 4 | 2014, mis en ligne le 15 mai 2014, consulté le 20 avril 2019. URL : http://journals.openedition.org/aes/286 ; DOI : 10.4000/aes.286

Ce document a été généré automatiquement le 20 avril 2019.

Centre de recherche LISAA (Littératures SAvoirs et Arts) 


\section{Herbert Spencer et la critique scientifique}

Taine, Hennequin, Brunetière

Marie Guthmüller

1 La présente contribution portera sur le rôle que joue la pensée d'Herbert Spencer pour la critique littéraire dite «scientifique» de la seconde moitié du XIx ${ }^{e}$ siècle en France. Les réflexions suivantes se situent dans le cadre d'une question plus large : comment cette critique se réfère-t-elle aux sciences de la nature pour étayer son exigence de ne plus figurer comme un genre littéraire, mais d'acquérir dorénavant, elle-même, le statut de science, voire de science positive? Pour y répondre, il faut retracer le dialogue noué par la critique littéraire avec des paradigmes qui marquent profondément la pensée scientifique de la deuxième moitié du siècle. Il sera donc question ici, tout d'abord, des conceptions novatrices d'une psychologie physiologique et expérimentale naissante, c'est-à-dire d'une "psychologie sans âme »1, comme le dira Théodule Ribot. Ensuite il s'agira de traiter de l'évolutionnisme qui, prenant comme point de départ le transformisme de Jean-Baptiste de Lamarck, deviendra, notamment avec la diffusion des œuvres de Charles Darwin, l'idée directrice des sciences de la vie. Pour la formation de ces deux paradigmes scientifiques en France, que ce soit pour celle d'une conception physiologique et expérimentale de la psychologie ou bien pour celle d'une notion évolutionniste de la vie, la pensée d'Herbert Spencer joue un rôle pivot.

2 Les protagonistes des réflexions suivantes seront les critiques Émile Hennequin et Ferdinand Brunetière. Tout en se trouvant l'un et l'autre dans le sillage de la critique scientifique dont le père fondateur est Hippolyte Taine ${ }^{2}$, ils n'ont de cesse de mettre l'accent sur leurs différences avec la pensée de leur précurseur. Les références respectives que font Hennequin et Brunetière - tout en se rapportant souvent à Spencer - à la psychologie physiologique et expérimentale ainsi qu'à la théorie de l'évolution, laissent entrevoir le dilemme dans lequel se trouve une critique littéraire qui cherche sa place dans le système des savoirs sur l'homme. Ce système connaît, en effet, des transformations rapides dans le climat du succès triomphant des sciences de la nature au 
cours du XIX ${ }^{e}$ siècle. Le dilemme de la critique littéraire, comme d'ailleurs celui des lettres et des sciences dites " morales $\|^{3}$ en général, se présente ainsi : comment se situer face à l'énorme succès des sciences physiques? Faut-il s'approcher le plus possible de leurs procédés, adopter leurs méthodes? Peut-on ainsi espérer consolider ses propres affirmations en s'appuyant sur la vérité que prétendent détenir ces sciences de la nature si prospères? Ou faut-il, au contraire, se renfermer sur des caractères spécifiques propres, revendiquer une forme de vérité différente ? Et, mieux encore, faut-il essayer de déconstruire les prétentions explicatives des sciences de la nature en réduisant la portée de leur validité?

3 Ces réflexions se situent dans le cadre des recherches que j'ai entreprises il y a quelques années. Dans Der Kampf um den Autor. Abgrenzungen und Interaktionen zwischen französischer Literaturkritik und Psychophysiologie, 1858-19104, je retrace les débats sur la nature de l'imagination poétique et sur l'acte de la création littéraire menés lors de la seconde moitié $\mathrm{du} \mathrm{xIx}^{\mathrm{e}}$ siècle. Ceux-ci opposent la critique littéraire dite scientifique à la psychologie physiologique et expérimentale naissante. Les lettrés d'un côté et les psychophysiologues et médecins de l'autre se disputent la capacité de définir ce qu'est l'auteur, - ou, autrement dit, le génie littéraire - et en quoi consiste l'acte de création. Dans ces controverses entre deux formes de savoir, différentes conceptions de ce qui est " scientifique » sont négociées. C'est la place des lettres et des sciences «non-physiques » dans le système des savoirs sur l'homme qui est en cause, tout comme la légitimité de la médecine et de la psychologie physiologique et expérimentale à s'approprier peu à peu les domaines anciennement réservés aux premières: l'âme, la société, la politique, l'histoire, les beaux-arts et, bien sûr, la littérature elle-même.

4 Le sort des écrits du jeune critique et journaliste Émile Hennequin montre le risque de faire double emploi encouru par la critique littéraire lorsqu'elle tente de trop se rapprocher des procédés et des prétentions explicatives des sciences physiques, notamment de celles de la médecine et de la psychologie physiologique. Dans La Critique scientifique parue en 1888, Hennequin redéfinit la critique littéraire en tant qu' " esthopsychologie » et la qualifie alors comme « un ordre de recherche où les œuvres d'art sont considérées comme les indices de l'âme des artistes et de l'âme des peuples " $^{5}$. En choisissant le terme "esthopsychologie", Hennequin démarque implicitement le concept d'«AEstho-Physiologie» proposé par Herbert Spencer dans Principles of psychology. L'ouvrage en deux volumes, publié en 1855 et réédité en 1872, a paru en France en 1874 et 1875 dans la traduction de Théodule Ribot et Alfred Espinas. Dans son ouvrage, Spencer introduit ce néologisme censé désigner un domaine intermédiaire entre la physiologie et la psychologie. L'« æsthophysiologie » entre en jeu quand les phénomènes nerveux sont à traiter comme phénomènes de conscience, à un moment clé de la nouvelle argumentation psychologique de Spencer :

La position de l'æsthophysiologie est tout à fait unique. Elle n'appartient ni au monde objectif, ni au monde subjectif, mais, prenant un terme de chaque, elle s'occupe de la corrélation des deux. Elle peut aussi bien être renfermée dans le domaine physique que dans le domaine psychique, et on peut la laisser où elle est, comme un trait d'union entre les deux. ${ }^{6}$

Cette formule, composée à partir d'" esthétique » et de "physiologie », Spencer ne la rattache donc pas spécialement à l'art, mais à l'aisthesis, c'est-à-dire à la théorie de la perception sensorielle. Hennequin semble alors jouer avec les différentes significations possibles d'« esthopsychologie ». Elle représente pour lui certainement une psychologie de l'art, mais dans sa conception de la critique scientifique, celle-ci étant effectivement 
étroitement liée à la théorie de la perception sensorielle ${ }^{7}$. Hennequin, en se référant à un terme qui définit pour l'un des pères fondateurs de la psychologie physiologique et expérimentale un domaine intermédiaire entre la physiologie et la psychologie, cherche$\mathrm{t}$-il sans doute surtout à profiter du caractère paradigmatique de celui-ci. Ce mot vise à la totalité, il qualifie une approche universaliste liant étroitement monde physique et monde moral. À la fin des années 1880, Spencer est désormais devenu en France l'un des partisans majeurs de la nouvelle psychologie, il représente une autorité intellectuelle incontournable ${ }^{8}$. Avec la notion d'«æsthophysiologie», il fournit à Hennequin un enchaînement tout trouvé grâce auquel celui-ci est à même d'étayer ses prétentions scientifiques. Dans La Critique scientifique, Hennequin utilise "esthopsychologie» en alternance avec «critique scientifique ». Il considère lui-même ce néologisme comme " incommode, disgracieux ", mais celui-ci dénotant d'une certaine teinture spencerienne se prête justement à merveille, à la fin des années 1990, à une modernisation et à une revalorisation scientifique de la critique littéraire.

6 C'est que Hennequin voit grand : il confère à l'« esthopsychologie » le statut de nouvelle supra-discipline renfermant psychologie, anthropologie et sociologie. Et c'est à ce titre qu'il la place à la tête des sciences morales et aussi des sciences physiques. Dans le cadre de cette approche, le texte littéraire, comme chaque œuvre d'art, est envisagé comme un document à partir duquel il devient possible non seulement d'analyser jusque dans ses moindres détails la disposition psychophysiologique de l'écrivain, mais aussi celle de ses lecteurs, et de tracer ainsi la psychologie d'un peuple en se basant sur ses œuvres d'art. L'esthopsychologue doit, selon Hennequin, procéder en trois étapes: d'abord l'analyse esthétique détaillée de l'œuvre et des émotions que les divers procédés littéraires utilisés suscitent chez le lecteur. Cette analyse prépare ensuite à l'analyse psychologique de l'auteur, pièce maitresse de cette nouvelle discipline. Plus les particularités esthétiques d'une œuvre sont nombreuses et importantes, plus les caractéristiques intellectuelles spécifiques de son auteur doivent l'être également. L'esthopsychologie est donc, pour Hennequin, une psychologie du spécifique et de la déviation. Son but consiste à établir ce en quoi l'artiste, en tant qu'artiste, se distingue des autres hommes et ce en quoi l'artiste, en tant qu'artiste spécifique, se distingue de ses confrères. La troisième et dernière étape est l'analyse sociologique de ses lecteurs. Une prédilection pour un certain style, pour certaines figures rhétoriques ou pour certains principes de forme indique que le lecteur doit avoir une disposition psychophysiologique proche de celle de l'écrivain ${ }^{10}$. Ce fait permet de prendre les œuvres d'art et les artistes comme point de départ d'une sociologie ou psychologie d'un peuple.

7 Si l'on compare la structure de la monographie d'Hennequin à celle de A System of Synthetic Philosophy ${ }^{11}$, ouvrage ambitieux en dix volumes dans lequel Spencer réunit, dès 1860, la plupart de ses écrits, quelques parallèles en ressortent. Une section introductive (Hennequin : Évolution de la critique, Spencer : First Principles) est suivie par une approche tripartite (Hennequin: Analyse esthétique, Analyse psychologique, Analyse sociologique; Spencer: The Principles of Biology, The Principles of Psychology, The Principles of Sociology), le tout se terminant avec une réflexion sur des questions de morale et d'éthique (Hennequin : La Critique et l'histoire, chapitre qui parle d'éthique ; Spencer : The Principles of Morality). Comme Spencer, Hennequin, en miniature mais non pas avec un enthousiasme moindre, s'applique non seulement à réorganiser les sciences sous un même principe, mais aussi à dépasser le clivage entre nature et culture en intégrant le domaine du moral tout comme celui du physique. 
Or Hennequin a compté sans son hôte, c'est-à-dire sans les représentants de la nouvelle psychologie physiologique. L'esthopsychologie, en revendiquant pour elle seule la capacité d'analyser scientifiquement à partir de leurs textes les dispositions psychophysiologiques des écrivains, s'avance si loin dans le domaine de la médecine et de la psychophysiologie que le jeune et ambitieux psychiatre Édouard Toulouse, en 1897, peut demander de bonne guerre dans la Revue scientifique :

Mais n'est-il pas paradoxal de soutenir que le biologiste, armé de ses instruments et pouvant à son aise interroger le sujet et le soumettre à toutes ses expériences, ne peut pas arriver à plus de vérité sur l'organisation de l'individu que l'artiste, simple littérateur, qui connaît seulement l'œuvre ? ${ }^{12}$

C'est justement cela, « interroger le sujet et le soumettre à toutes ses expériences », que Toulouse « armé de ses instruments » avait fait lui-même deux ans auparavant. En 1896, il avait publié les Enquêtes médico-psychologiques sur les rapports de la supériorité intellectuelle avec la névropathie ${ }^{13}$. Ces enquêtes, entreprises entre autres sur Émile Zola, avaient été menées dans le nouveau Laboratoire de psychologie physiologique de la Sorbonne récemment fondé par Alfred Binet et Henri Beaunis. Outre des «mental tests » récemment importés des États-Unis ${ }^{14}$ ainsi que des tests de réactivité d'après le psychologue Wilhelm Wundt de Leipzig et des examens graphologiques, Zola fut soumis à d'étendus examens anthropométriques et surtout craniologiques.

10 La critique de Toulouse semble d'autant plus légitime que Hennequin, dans sa monographie, s'efforce d'utiliser constamment un vocabulaire emprunté à la nouvelle psychologie expérimentale et physiologique. Par ailleurs, il y affirme lui-même que l'esthopsychologie, censée "tirer de l'œuvre d'art l'image de l'esprit», doit figurer comme substitut temporaire de la neuroanatomie dont seules les futures recherches histologiques confirmeront définitivement les résultats :

[...] on peut imaginer tels progrès de la science des rapports de la pensée avec le cerveau qui permettront d'étayer l'hypothèse psychologique sur l'organisation mentale d'un artiste, par une hypothèse physiologique sur la confirmation de son cerveau; une supposition de ce genre pourra même être confirmée par l'examen histologique de l'encéphale qui en aura été l'objet. De pareilles vérifications, si elles sont favorables, donneront à nos analyses critiques une valeur absolue. ${ }^{15}$

11 La critique littéraire, en se rapprochant trop du vocabulaire, des méthodes et des objectifs de connaissance d'une science de la nature en plein essor, court de toute évidence le risque de se rendre superflue, ou du moins intérimaire et provisoire.

Ferdinand Brunetière, doyen de la rhétorique universitaire, de l'histoire littéraire et de la critique, est à l'apogée de sa carrière dans les années 1880 et 1890. Il semble avoir été conscient du risque couru par son jeune confrère, risque qu'il voit déjà s'amorcer dans les écrits d'Hippolyte Taine avec lesquels il entretient un rapport ambigu. Dans un compte rendu de «La Critique scientifique, par M. Émile Hennequin », paru en 1888 dans La Revue des Deux Mondes, il pose la question rhétorique suivante :

[...] au lieu de « juger » l'art, quel avantage voit-on à ce que la critique, en devenant "scientifique», devienne une branche de la psychologie, la psychologie des "géniaux ", selon l'expression de M. Hennequin, lisez, en plus clair, quelque chose d'analogue, d'accessoire et de subsidiaire à la pathologie mentale ?16

13 Il n'y a pas de doute sur la réponse suggérée par Brunetière: une telle démarche ne présente pas le moindre «avantage». Si la critique littéraire ne veut pas devenir un élément « accessoire et subsidiaire à la pathologie mentale » et ainsi contribuer peu à peu à sa propre abolition, elle doit rebrousser chemin, prendre ses distances avec la 
psychologie physiologique tentée par le déterminisme, et se définir autrement. Elle doit, et Brunetière n'a de cesse de le souligner, se souvenir de son ancienne mission et devenir de nouveau une critique, un art supposé juger les œuvres et non les traiter seulement comme des signes de quelque chose qui leur est extérieur.

Mais cette redéfinition de la critique littéraire pose problème car il s'agit à la fois, pour Brunetière comme pour Taine et Hennequin, de baser leur discipline sur des critères qui leur permettent de se distinguer des considérations esthétiques subjectives et arbitraires. C'est en effet contre une critique dont les jugements dérivent du «caprice » et de la "fantaisie $»^{17}$ que la critique scientifique se positionne. À l'instar de ses confrères, Brunetière recherche bel et bien l'objectivité. Comment alors appuyer ses propres jugements esthétiques et sur quoi les baser? L'argumentation de Brunetière séduit par sa simplicité : il faut fonder la critique littéraire, comme l'a entrepris Nicolas Boileau plus de deux siècles auparavant, sur la nature. L'histoire littéraire montrerait, et en cela Taine aurait eu raison, des analogies avec l'histoire naturelle - et ce sont sur ces analogies-là que le critique peut fonder ses considérations ${ }^{18}$.

C'est justement ici que la théorie de l'évolution entre en jeu - et avec elle de nouveau la pensée d'Herbert Spencer. Après avoir présenté une ébauche des débats menés par une critique littéraire se voulant scientifique avec la psychologie physiologique et expérimentale naissante, nous aborderons maintenant la manière dont elle entre en dialogue avec le deuxième paradigme scientifique en question. En effet, dans la seconde moitié du XIX ${ }^{e}$ siècle, la théorie de l'évolution révolutionne non seulement l'ancienne histoire naturelle, mais devient, notamment par le biais de l'énorme succès de l'œuvre de Spencer, le point d'appui prédominant de la plupart des sciences contemporaines. Spencer a longtemps été considéré comme celui qui aurait appliqué la pensée darwinienne et évolutionniste aux sociétés humaines - avec des conséquences néfastes, atteignant leur point culminant dans la politique raciale du fascisme allemand; d'où les expressions " darwinisme social ", « Sozialdarwinismus » ou "Social Darwinism » très en usage dans les sciences humaines à la fin du $\mathrm{xx}^{\mathrm{e}}$ siècle $^{19}$. Mis à part le fait que Spencer, influencé par des conceptions pré-darwiniennes de l'évolution biologique comme celles de Lamarck, ait adopté un point de vue évolutionniste avant même la parution de L'Origine des espèces en $1859^{20}$, il est important de souligner qu'il n'étend pas une théorie d'origine biologique au domaine social. Au contraire, dès ses premiers écrits datant des années 1850, il développe, sur fond de différents courants philosophiques et scientifiques apparus au milieu du XIX ${ }^{\mathrm{e}}$ siècle (lesquels constituent également la base épistémologique de l'œuvre darwinienne), un point de vue évolutionniste global. il le juge valable dans tous les domaines du savoir, que ce soit dans la géologie et la biologie ou dans la psychologie et la sociologie. D'après lui, le développement de l'univers tout entier consiste en un changement de l'homogène à l'hétérogène :

The advance from the simple to the complex, through a process of successive differentiations, is seen alike in the earliest changes of the Universe to which we can reason our way back, and in the earliest changes which we can inductively establish; it is seen in the geologic and climatic evolution of the Earth, and of every single organism on its surface; it is seen in the evolution of Humanity, whether contemplated in the civilized individual, or in the aggregation of races; it is seen in the evolution of Society in respect both of its political and economical organization; and it is seen in the evolution of all those endless concrete and abstract products of human activity which constitute the environment of our daily life. From the remotest past which Science can fathom, down to the novelties of yesterday that in 
which Progress essentially consists, is the transformation of the homogeneous into the heterogeneous. ${ }^{21}$

à une théorie de l'évolution marquée encore essentiellement par l'ancienne histoire naturelle. Pour étayer ses réflexions sur les « caractères primordiaux » des œuvres d'art, il s'en remet à la Philosophie anatomique d'Étienne Geoffroy Saint-Hilaire. Hennequin et Brunetière appuient, quant à eux, leurs réflexions concernant les tâches, les méthodes, le sujet de recherche et le but de la critique littéraire sur les théories de l'évolution darwinienne et spencérienne alors en plein essor ${ }^{24}$. Plusieurs approches sont spécialement intéressantes dans ce contexte. Il y a d'abord le tri que font Hennequin et Brunetière parmi des éléments fondamentaux de la théorie de l'évolution. Ensuite, le fait qu'ils manient le pouvoir explicatif de celle-ci comme un tout. Et enfin leurs hypothèses relatives à la possibilité d'appliquer à l'histoire des êtres humains et de leurs acquis culturels une thèse qu'ils considèrent, eux, comme prouvée dans le domaine de l'histoire naturelle. C'est ici que nous allons retrouver le dilemme qui apparait déjà dans les débats portant sur les analyses psychophysiologiques des écrivains : qui ont opposé les critiques littéraires aux médecins et psychologues : comment prendre part à l'autorité des sciences positives sans se faire dévorer par elles ? Comment devenir « scientifique » sans hâter sa propre abolition?

Cette fois-ci, Hennequin comme Brunetière semblent déployer une stratégie argumentative analogue, bien que ce soit encore Brunetière qui, en fin de compte, se révélera le plus prudent et le plus habile. Brunetière et Hennequin basent l'un comme l'autre de larges parties de leur œuvre de critique et d'historien de la littérature sur la théorie de l'évolution. Ils citent Charles Darwin, Herbert Spencer et, en ce qui concerne Brunetière, également le biologiste et philosophe allemand Ernst Haeckel. Toutefois ils développent tous les deux en même temps, tout en continuant à recourir à des énoncés principaux de la théorie même, une argumentation qui leur permet d'extraire de l'histoire naturelle un domaine spécifiquement humain dans lequel règnent des lois différentes. Cela paraît convaincant dans la logique interne de l'œuvre de Brunetière. Alors qu'il considère la nouvelle psychologie physiologique et expérimentale comme déterministe et réductionniste, l'histoire naturelle et la physiologie du développement lui semblent offrir la possibilité de rétablir les concepts d'individualité, de libre arbitre et de génie - et ainsi de créer un domaine de recherches exclusivement réservé aux hommes de lettres. En revanche chez Hennequin, partisan de la nouvelle psychologie, dont il veut par ailleurs dépasser la portée avec son "esthopsychologie», les contradictions argumentatives semblent être plus saillantes.

Venons-en d'abord à Hennequin. En ce qui concerne les thèses ayant trait à l'histoire naturelle et à la biologie, il semble se montrer plus prudent qu'avec celles de la 
psychologie physiologique. Il souligne en effet qu'il ne se borne pas, comme l'aurait fait, selon lui à tort, Hippolyte Taine avec les théories du milieu et de l'hérédité, à simplement transférer les thèses des sciences naturelles aux domaines du social et de l'art. Il s'agirait plutôt, pour l'esthopsychologie, qu'elle vérifie elle-même la portée de ces hypothèses-là. C'est ainsi que Hennequin inverse les suppositions des théories du milieu et de la race si chères à Taine. D'après Hennequin, l'homme, comme d'ailleurs chaque être vivant, s'adapterait le moins possible à son milieu, afin d'épargner ses forces. Il chercherait à atteindre le plus d'indépendance possible de son milieu physique et social et s'adapterait essentiellement de manière défensive. D'après Hennequin et contrairement à Taine, ce n'est donc pas le milieu qui marque l'artiste, mais c'est l'artiste qui produit seulement un certain milieu $^{25}$.

De plus, Hennequin se réfère, contre Taine et la théorie du milieu, à l'idée d'Herbert Spencer selon laquelle le développement de tout organisme consiste en un changement de l'homogène à l'hétérogène, changement valable au niveau de l'humanité tout entière. Comme on l'a vu, Spencer développe cette idée cruciale pour sa conception du progrès et de l'évolution, à partir des années 1850 . Nous la trouvons dès 1871 en France, date de parution de la traduction française par Emile Cazelles des First Principles (1860-1862): "Telle que nous la comprenons maintenant, l'évolution peut se définir comme un changement d'une homogénéité incohérente en une hétérogénéité cohérente, à la suite de la dissipation du mouvement et de l'intégration de la matière. ${ }^{26}$ Hennequin n'attribue pas cette analogie entre culture et nature au fait que le développement de l'homme et de la nature soit soumis à une même loi de la nature. Ce ne sont, d'après lui, que les institutions sociales qui, en figurant comme « défenses » contre la nature, ont permis peu à peu une différenciation entre les hommes. C'est depuis sa socialisation que l'espèce humaine constitue à l'intérieur du règne animal un règne à part dans lequel s'appliquent des lois différentes - et c'est seulement ainsi qu'un développement historique vers l'indépendance et vers l'hétérogène devint possible, un changement qui vit les hommes différer de plus en plus les uns des autres. Le passage à l'hétérogène devient de la sorte, $\mathrm{du}$ point de vue de l'esthopsychologue, un acquis culturel ${ }^{27}$. Hennequin peut ainsi considérer ce principe de la théorie de l'évolution comme valable pour tous les domaines de la civilisation humaine, y compris l'histoire littéraire, sans abandonner le rôle prédominant de l'esthopsychologie au sommet de la pyramide des connaissances.

Hennequin réussit à faire encore mieux. En se référant à deux principes centraux de la théorie darwinienne de l'évolution, «la variation spontanée » ainsi que "la répétition », et en les identifiant aux deux principes sociologiques de Gabriel Tarde, «l'invention » et « l'imitation », il parvient à rétablir " scientifiquement » le génie littéraire et à le déclarer sujet de recherche de l'esthopsychologie. C'est le principe de la « variation spontanée » ou de "l'invention" qui confère à un artiste, ou bien à un héros, une disposition psychophysiologique spécifique qui le rend capable de rassembler autour de lui, via le principe de la «répétition" ou de "l'imitation», tous ceux dont la disposition psychophysiologique correspond à la sienne. ${ }^{28}$ C'est ainsi que l'artiste crée son public et le héros le cercle de ses partisans. Hennequin souligne que la loi sociologique de l'imitation est, dans le règne humain, beaucoup plus importante que le principe de l'hérédité biologique. Il combine les théories de Darwin et de Tarde de telle manière que, dans toutes les questions d'ordre sociologique, l'importance est obligatoirement donnée à la critique scientifique. Il n'y a que l'esthopsychologie, flanquée d'une psychologie des héros, qui soit qualifiée pour porter son attention sur ces individus rares et hors du 
commun, ceux-ci étant, grâce à leur disposition psychophysiologique spécifique, seuls capables de créer des organismes sociaux.

C'est pour cette argumentation-là, pour sa prise de distance avec Taine et pour sa réfutation des théories de l'hérédité et du milieu appliquées aux artistes, que Ferdinand Brunetière, dans son compte rendu de 1888 , loue son jeune collègue tant critiqué pour son flirt dangereux avec la psychophysiologie ${ }^{29}$. Comme Hennequin, il soulignera, en se référant lui aussi aux "variations spontanées» de Darwin, la singularité et l'indépendance des auteurs littéraires. C'est dans ses cours sur L'Évolution des genres dans l'histoire de la littérature, donnés en 1889 à l'École normale que Brunetière affirme que les analogies entre une œuvre d'art et une société ne proviendraient en effet pas de l'influence du milieu sur l'artiste, mais plutôt du fait que l'œuvre crée son public. Et en se référant, comme Hennequin, à Herbert Spencer, Brunetière approuve dans son ensemble le principe d'une évolution de l'homogène à l'hétérogène et postule lui-même une différenciation croissante des genres littéraires: "Sans doute, la différenciation des genres s'opère dans l'histoire comme celle des espèces de la nature, progressivement, par transition de l'un au multiple, du simple au complexe, de l'homogène à l'hétérogène, grâce au principe qu'on appelle de la divergence des caractères [...]. . ${ }^{30}$

Brunetière considère la théorie de l'évolution comme étant la base fondamentale de tous les domaines du savoir de son époque. De ce fait acquis, il résulterait la nécessité, voire l'inévitabilité d'éprouver désormais la fécondité de cette théorie pour la critique littéraire. Mais dans un même temps, il n'oublie pas de souligner à plusieurs reprises que la critique littéraire ne gagne rien en prétendant elle-même accéder à un statut de science. Dans ses cours sur l'évolution des genres littéraires, il qualifie, en toute logique, la critique de quatrième genre littéraire aux côtés de la poésie, du drame et de la prose.

En écrivant une histoire littéraire reposant sur la théorie de l'évolution, Brunetière espère pouvoir éviter toute tendance à l'énumération chronologique et rendre visibles des liens généalogiques entre les œuvres. Or, son recours à la théorie de l'évolution doit lui permettre non seulement de donner un fil conducteur à l'histoire littéraire, mais surtout de développer des critères considérés comme objectifs pour la classification et pour le jugement des œuvres. Cela lui permettra ainsi de consolider, comme on l'entrevoit, une compréhension anti-moderniste de ce qu'est la littérature. Il admet par exemple que les genres littéraires, à l'instar des espèces des règnes végétal et animal, apparaissent, puis atteignent leur perfection, pour enfin décliner et mourir. Il suppose, en se référant à des concepts comme la " concurrence vitale ", la « persistance du plus apte » et la «sélection naturelle», que le processus de transformation auquel sont soumis les genres littéraires présente de nombreuses analogies avec celui de la transformation des espèces :

Et, dès à présent, si l'apparition de certaines espèces, en un point donné de l'espace et du temps, a pour effet de causer la disparition de certaines autres espèces; ou encore, s'il est vrai que la lutte pour la vie ne soit jamais plus âpre qu'entre espèces voisines, les exemples ne s'offrent-ils pas en foule pour nous rappeler qu'il n'en est pas autrement dans l'histoire de la littérature et de l'art ? $^{31}$

Comme Hennequin dans La Critique scientifique, Brunetière, dans L'Évolution des genres, se fonde sur le principe des "variations spontanées", considérées comme éléments déclencheurs des transformations de l'histoire littéraire: "[...] il n'y a rien de plus conforme à la doctrine de l'évolution que d'insister sur cette cause modificatrice des genres, puisqu'à vrai dire, selon l'Origine des Espèces, ce serait l'idiosyncrasie qui serait le 
commencement de toutes les variétés. ${ }^{32}$ Il est ainsi à même de consolider "scientifiquement » une idée qui lui est chère : celle du génie littéraire, inexplicable par les sciences de la nature, spécialement par la psychophysiologie. Puisque, d'après Darwin, ce sont toujours des idiosyncrasies qui se trouvent au départ de la formation des variétés, et qui sont décisives pour la transformation des espèces, Brunetière peut donc placer le génie idiosyncratique, singulier et inexplicable, au départ de la transformation des genres littéraires. C'est ainsi que la théorie de l'évolution, considérée comme fleuron du pouvoir explicatif universel et inaltérable des sciences de la nature, fournit elle-même la preuve de l'existence de l'individuel, du contingent et de l'accidentel. Elle rend possible la constitution d'un espace propre au domaine de l'esprit, espace où peut s'exercer le libre arbitre de l'homme, et elle peut donc être invoquée contre les prétentions universalistes de la psychophysiologie. Du point de vue de Brunetière, la théorie de l'évolution peut de la sorte devenir le garant d'un domaine indépendant de l'esprit humain et d'un sujet de recherche exclusivement réservé à la critique littéraire.

Toutefois, Brunetière semble avoir été conscient du fait que son argumentation se mordait la queue. Le génie est sauvé, le sujet de recherche propre à la critique littéraire consolidé - mais cela seulement au prix d'une dépendance des résultats de recherche visà-vis des sciences de la nature. Et s'il n'avait pas fait mieux que son jeune confrère ? S'il avait, lui aussi, contribué à l'abolition graduelle de la critique littéraire - et des sciences morales en général ? Il n'a certainement pas échappé à Brunetière qu'il a seulement pu rétablir la valeur des sciences morales en faisant appel aux sciences physiques.

C'est dans ces circonstances que Brunetière souligne de nouveau l'indispensabilité d'un domaine de recherche et d'une méthodologie propres à la critique littéraire. Taine n'aurait pas entrevu que la condition essentielle de l'homme se trouvait justement dans sa différence avec l'animal, car le destin de son espèce se distinguait depuis des milliers d'années de celui des espèces du règne animal. L'argumentation de Brunetière devient plus nuancée. En se portant de nouveau sur la psychocritique de Taine et de Hennequin, il a maintenant recours aux principes de la rhétorique. Il souligne le fait que la disposition psychophysiologique d'un écrivain ne doit pas obligatoirement s'exprimer dans son œuvre, celui-ci pouvant très bien décider de ne pas laisser filtrer sa propre personnalité dans ses textes. Comme l'orateur, il peut opérer un choix entre différents discours, sa manière de s'exprimer n'étant jamais simplement naturelle. L'homme, être vivant doté du langage, et notamment l'écrivain, homme doté en particulier de la faculté de connaître le fonctionnement du langage et de le manier, est plus complexe que ne se l'imaginaient les sciences de la nature ${ }^{33}$.

À la fin des années 1890, à la suite de sa conversion au catholicisme, Brunetière reprend finalement confiance en lui ainsi que dans les sciences morales. Son argumentation change de ton et semble gagner en solidité et en force de persuasion. Un point de repère principal devient alors pour lui la théorie de l'« Inconnaissable », formulée par Herbert Spencer dans la première partie des Premiers Principes, précédant celle, majeure, intitulée "Le Connaissable $~^{34}$. Dans un climat de scepticisme croissant envers l'espoir mis dans le progrès des sciences, cette thèse, développée par Spencer déjà quelques décennies auparavant, devient d'une manière générale très en vogue en France autour de $1900^{35}$. Brunetière essaie, lui, de la concilier avec celle de la relativité de la connaissance d'Auguste Comte et développe, en se basant sur l'une comme sur l'autre, sa propre théorie fondamentale de la connaissance. Il s'agit notamment de reformuler la relation entre science et religion et, ce faisant, de résoudre leur antagonisme. Brunetière 
détermine les liens entre les deux domaines ainsi que la répartition des compétences entre eux en basant les conditions de possibilité de la science sur l'inconnaissable :

À l'extrémité du domaine où la «Science» est souveraine, la théorie de l'Inconnaissable a dressé la borne qu'on pourra déplacer, mais qu'on ne renversera pas, ou à laquelle, quand on croira l'avoir renversée, on ne continuera pas moins de se heurter toujours. Elle a posé aussi la borne qui sépare le domaine de la science du domaine de la morale ou de la religion ${ }^{36}$.

Dans ce contexte, le recours de Brunetière à la théorie de l'évolution se modifie de manière fondamentale. Brunetière continue bel et bien de s'appuyer sur Spencer comme sur Darwin, mais désormais dans le but principal de réfuter l'idée du progrès et de donner une base au dogme du péché originel. Il ne considère toutefois plus la théorie de l'évolution comme l'œuvre exclusive des sciences de la nature. Il commence, en effet, à recenser tout ce que doit cette théorie aux sciences morales, notamment à la philosophie, aux lettres et à la théologie. Comme l'avait déjà fait avant lui le zoologiste allemand Ernst Haeckel, il attribue la "doctrine évolutive» au livre de la Genèse et souligne son caractère finalement téléologique. Et il lui octroie, en plus, un caractère fictionnel : « [...] et ce que j'avoue moi-même, c'est que la Descendance de l'homme de Darwin, ou l'Histoire nouvelle de la Création du professeur Haeckel, ne sont, de leur vrai nom, que des romans scientifiques. $»^{37}$

31 L'argumentation de Brunetière est, à mon sens, très habile. À défaut de contester la théorie de l'évolution qui est devenue, à la fin du siècle, la représentante de la pensée scientifique par excellence et la pierre de touche du renouveau catholique, Brunetière utilise cette théorie comme pilier de ses propres hypothèses. Mais il développe, avant de disparaître dans le magasin d'accessoires du XIX ${ }^{e}$ siècle, une figure argumentative encore plus originale et décisive. Il ne revendique pas pour la critique littéraire, comme l'ont fait ses confrères Taine et Hennequin, un statut de science positive, il ramène plutôt les succès d'une des théories scientifiques les plus importantes et célèbres aux acquis des lettres - et situe ainsi son pouvoir explicatif sur le même plan. Dans le débat des deux cultures qui se prolonge jusqu'à nos jours, cela paraît être une stratégie argumentative bien actuelle.

Durant la deuxième moitié du XIX ${ }^{e}$ siècle en France, le rôle joué par la pensée d'Herbert Spencer à l'intérieur des débats récents entre sciences morales et sciences physiques est crucial. Les références multiples qu'une critique littéraire se voulant scientifique entretient avec la pensée spencérienne en forment un aspect fondamental. Que ce soit Émile Hennequin ou Ferdinand Brunetière, tous deux se réclament de Spencer pour des points clés de leur argumentation. Notamment quand il s'agit de se démarquer de Taine, père fondateur de la critique scientifique dont l'approche aux œuvres littéraires, considérée de plus en plus souvent comme matérialiste et réductionniste, commence à tomber en discrédit à la fin du siècle. Le rôle de Spencer est essentiel à la fois pour les références que Hennequin et Brunetière font à une conception physiologique et expérimentale de la psychologie et pour celles qu'ils font à la pensée évolutionniste. Il s'agit là, comme on a vu, de deux paradigmes scientifiques qui se prêtent spécialement à une satisfaction des revendications épistémologiques d'une « science de la littérature » en devenir. Désormais, l'œuvre de Spencer semble être, à la fin du siècle, un pilier plus approprié à une telle entreprise que celui de Taine, l'approche du philosophe anglais prêtant beaucoup moins le flanc aux attaques des adversaires. Son aptitude à recueillir un large consensus réside très probablement dans le fait que Spencer, dès le début, réserve une place à ce qui ne se laisse pas expliquer scientifiquement et qu'il ne conçoit pas le 
domaine de la culture en analogie avec celui de la nature, mais qu'il les conçoit tous deux comme des unités indissociables soumises d'office aux mêmes lois.

Figure : Différenciation croissante de la littérature française au cours des siècles, développement de l'homogène à l'hétérogène d'après la théorie d'Herbert Spencer, voir : Émile Hennequin, La Critique scientifique, op. cit., p. 125.

\section{LITTÉRATURE. FRANÇAISE}

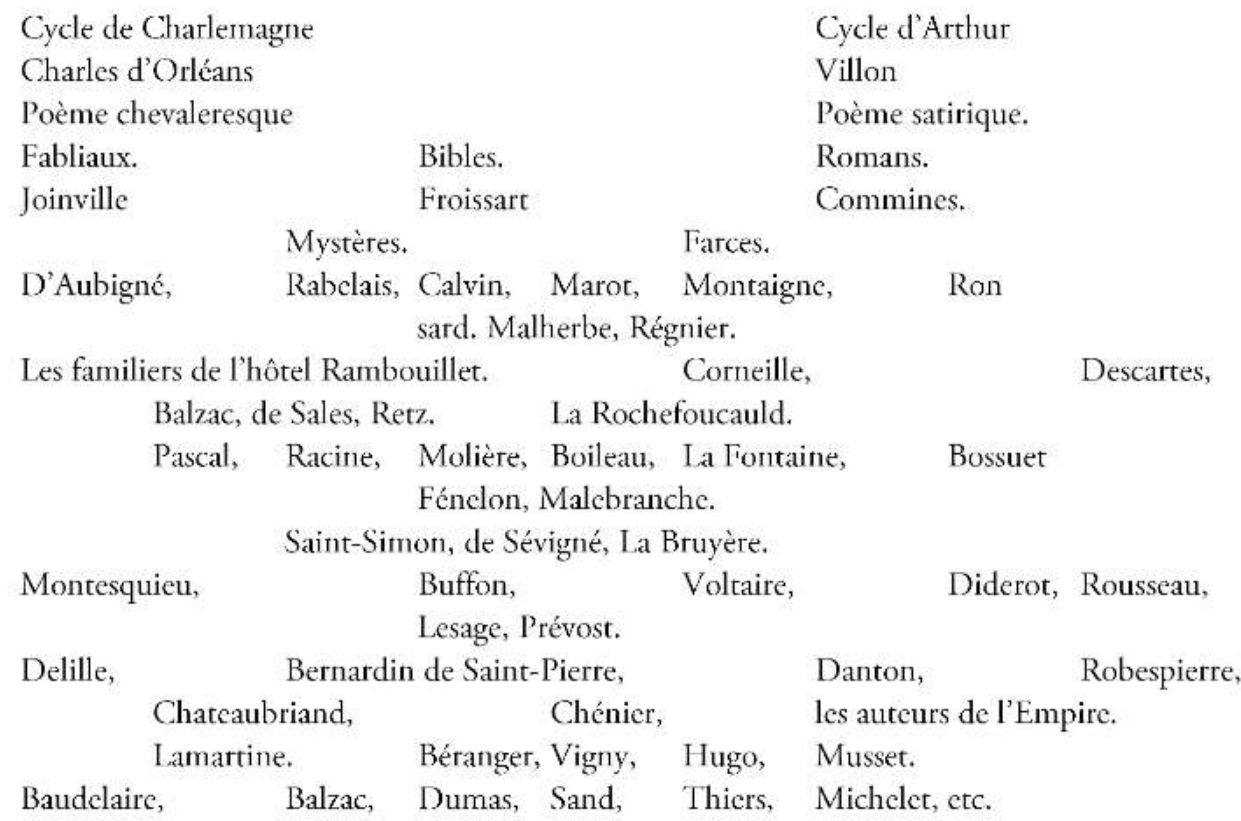

\section{NOTES}

1. C'est en 1879, dans la préface de La Psychologie allemande contemporaine (école expérimentale) (Paris, Baillière, 1879), que Théodule Ribot revendique explicitement « une psychologie sans âme » (p. VIII). Il développe les principes de cette nouvelle psychologie à partir de 1870, tout d'abord dans La Psychologie anglaise contemporaine, école expérimentale (Paris, Ladrange, 1870) qui consacre un long chapitre à l'œuvre de Spencer: «La psychologie dont il s'agit ici sera donc précisément expérimentale : elle n'aura pour objet que les phénomènes, leurs lois et causes immédiates ; elle ne s'occupera ni de l'âme ni de son essence, car cette question, étant en dehors de la vérification, appartient à la métaphysique. » («Introduction », p. 29.)

2. En ce qui concerne les références d'Hippolyte Taine même à Herbert Spencer, celles-ci sont surtout importantes dans le domaine de la philosophie, voire de la psychologie. Taine critique ne se réfère pratiquement pas à Spencer. Par contre en matière de psychologie, Taine n'est pas seulement un grand divulgateur mais aussi un concurrent de son collègue anglais. Il revendique ainsi, en 1873, la priorité sur la théorie du choc nerveux que Spencer réclame pour lui. C'est, écrit Taine «trait pour trait ce que j'ai écrit dans l'Intelligence, I, p. 202 à 219. [...] Ce n'est pas moi qui l'ai suivi car rien de tout cela ne se trouvait dans la première édition de sa psychologie. » Taine se 
révolte contre l'affirmation de Spencer, dans la seconde préface aux Principles of Psychology (Londres, Williams and Norgate, 1870-1871), selon laquelle De l'Intelligence (Paris, Hachette, 1870) aurait «fait connaître en France quelques-unes de ses maitresses conceptions »; après cela les revues anglaises feraient De l'Intelligence « une simple imitation, une transcription française des théories anglaises » («Lettre à M. Th. Ribot, 11 janvier 1873 », Taine, sa vie et sa correspondance, $\mathrm{t}$. III, L'Historien, Hachette, 1905, p. 215-218).

3. Tout au long du $\mathrm{XIX}^{\mathrm{e}}$ siècle, à une époque où l'idée de sciences "de l'homme " (appelées aujourd'hui sciences "humaines» ou "sociales») commence seulement à se développer, les «sciences morales » sont une première expression du projet d'organiser toutes les sciences «non physiques » et de les mettre au service du progrès humain. Le projet de réaliser une classification des savoirs anciens et nouveaux sur l'homme et sur la société afin de les représenter au sein d'institutions officielles remonte à la fin du XVIII ${ }^{\mathrm{e}}$ siècle, notamment à la création d'une "classe de sciences morales et politiques » au sein de l'Institut de 1795, sous la Révolution. Les « sciences morales » visant à la fois la psychologie, l'éthique, la politique et l'histoire, l'expression fait ressortir une forte empreinte métaphysique qui lie en fin de compte l'âme humaine à une origine divine et à ses devoirs envers Dieu. Julien Vincent en entreprend une première esquisse dans "Les "sciences morales" : de la gloire à l'oubli ?", La Revue pour l'histoire du CNRS [En ligne], n 18, 2007 (mis en ligne le 3 octobre 2009, consulté le $1^{\text {er }}$ mars 2012. URL: http://histoirecnrs.revues.org/4551, sans toutefois en souligner l'origine et sans retracer les problèmes complexes qui en résultent.

4. Marie Guthmüller, Der Kampf um den Autor. Abgrenzungen und Interaktionen zwischen französischer Literaturkritik und Psychophysiologie, 1858-1910, Tübingen, Francke, 2007. Le titre peut se traduire comme suit: «La lutte pour l'auteur. Démarcations et interactions entre critique littéraire et psychologie physiologique, 1858-1910».

5. Émile Hennequin, La Critique scientifique, Perrin, 1888, p. 3 : «[...] celui [le vocable propre] d'esthopsychologie pourrait convenir à un ordre de recherche où les œuvres d'art sont considérées comme les indices de l'âme des artistes et de l'âme des peuples; mais ce mot est incommode, disgracieux ; nous nous excusons de l'employer parfois et nous le remplacerons le plus souvent par le terme critique scientifique que nous opposons à critique littéraire dans un sens à préciser. »

6. Herbert Spencer, Principes de psychologie, trad. par Th. Ribot et A. Espinas d'après la deuxième édition de 1872, 2 vol., Paris, Ladrange, 1874-1875, vol. I, p. 130. Le texte original se présente ainsi : "AEsthophysiology has a position that is entirely unique. It belongs neither to the objective world nor the subjective world; but taking a term from each, occupies itself with the correlation of the two. It may with as much propriety be included in the domain of physical science as in the domain of psychical science; and must be left where it stands, as the link between them. ", Herbert Spencer, Principles of Psychology [1855/1872], vol. I (A System of Synthetic Philosophy, vol. IV), New York, Appleton, 1873, p. 130.

7. Hennequin cherche un compromis entre l'hypothèse d'une déterminabilité des sentiments esthétiques, où il distingue ceux de l'envie et ceux de la non-envie, comme celle qui imprègne par exemple l'esthétique d'un Charles Féré, et l'hypothèse d'une "satisfaction désintéressée » au sens de Kant. Dans ce contexte, il s'oriente vers une définition du sentiment esthétique comme «faible », telle qu'entreprise dans les Principes de psychologie de Spencer (à l'opposé de la douleur, qui est un sentiment «fort »), mais il émet la critique selon laquelle Spencer n'aurait pas fondé celle-ci suffisamment. (Voir Hennequin, La Critique scientifique, op. cit., p. 26 sqq.)

8. Voir surtout Daniel Becquemont et Laurent Mucchielli : La Cas Spencer. Religion, science et politique, Paris, PUF, 1998.

9. Hennequin, La Critique scientifique, op. cit., p. 3.

10. «[...] les admirateurs d'une œuvre d'art doivent posséder une organisation psychologique analogue à celle de son auteur, et l'âme de ce dernier étant connue par l'analyse, il sera légitime 
d'attribuer à ses admirateurs les facultés, les défauts, les excès, toutes les particularités saillantes de l'organisation mentale qui lui aura été reconnue. » Ibid., p. 139.

11. Ce n'est qu'en 1911 que les œuvres réunis de Spencer commencent effectivement à paraître sous ce titre conçu par l'auteur; Herbert Spencer, A System of synthetic philosophy, Londres, Williams and Norgate, 1911.

12. Édouard Toulouse, «La critique scientifique ", Revue scientifique, $\mathrm{n}^{\circ} 22,1897$, p. 678-684, p. 681.

13. Id., Enquête médico-psychologique sur les rapports de la supériorité intellectuelle avec la névropathie. Vol. I : Introduction générale : Émile Zola, Société d'éditions scientifiques, 1896. Voir sur cette étude le chapitre «Der psychologische Blick auf den literarischen Autor » (« Le regard psychologique sur l'auteur littéraire »), Guthmüller, Der Kampf um den Autor, op. cit., p. 152-182.

14. Ce fut James MacKeen Catell, premier professeur de psychologie expérimentale aux ÉtatsUnis depuis 1887, qui publia en 1890 « Mental tests and measurements », article qui est considéré aujourd'hui comme un travail pionnier en matière de recherche sur l'intelligence (« Mental tests and measurements ", Mind, $\mathrm{n}^{\circ}$ 15, 1890, p. 373-381). C'est Alfred Binet qui continuera cette sorte de recherche au début $\mathrm{du} \mathrm{xx}^{\mathrm{e}}$ siècle, toutefois en appuyant ses tests sur l'étude de facultés mentales « supérieures » comme la mémoire, l'imagination et l'attention.

15. Hennequin, La Critique scientifique, op. cit., p. 89.

16. Ferdinand Brunetière, "La Critique scientifique, par M. Émile Hennequin. Paris, 1888 ; Perrin », Revue des Deux Mondes, $n^{\circ}$ 88, juillet 1888, p. 213-226.

17. Ferdinand Brunetière, L'Évolution des genres dans l'histoire de la littérature: leçons professées à l'École normale supérieure [1889], vol. I, «Introduction: L'évolution de la critique depuis la Renaissance jusqu'à nos jours ", Hachette, 1890, p. 30. Brunetière se réfère ici à Anatole France qui avait exigé du critique littéraire de «suivre ses goûts, ses fantaisies et même son caprice, à la condition d'être toujours vrai, sincère et bienveillant "; $c f$. Anatole France, La Vie littéraire, Paris, Calmann-Lévy, 1895, p. XIX.

18. Brunetière, L'Évolution des genres, op. cit., p. 16 sqq.

19. L'expression cherche généralement à la fois à consolider la force explicative générale de la théorie darwinienne dans le règne des plantes et des animaux et à critiquer comme inadmissible son application dans le domaine de l'homme et de ses sociétés.

20. Herbert Spencer, Social Static, or the Conditions essential to human happiness specified, and the first of them developed, Londres, John Chapman, 1851.

21. Herbert Spencer, "Progress: Its Law and Cause ", The Westminster Review, n 67, 1857, p. 445-485, p. 465.

22. En ce qui concerne cette évaluation inexacte de Taine qui persiste jusqu'à nos jours, je me permets de renvoyer au premier chapitre de Guthmüller, Der Kampf um den Autor, op. cit., ainsi qu'à Marie Guthmüller : "Procédés empiriques et savoir esthétique. Hippolyte Taine fondateur de la "critique scientifique" et de la "psychologie expérimentale" ", Jacqueline Lichtenstein, Carole Maigné et Arnauld Pierre (dir.), Vers la science de l'art. L'esthétique en France 1857-1937, Paris, Presses Universitaires Paris Sorbonne, 2013, p. 47-60.

23. En 1904, dans la Revue des Deux Mondes, le philosophe Gaston Rageot décrit avec pertinence cette place occupée par Spencer: «Chez nous, Spencer a réussi à la fois par le détail et par l'ensemble: sa psychologie a suscité une école qui le respecte encore et qui se borne le plus souvent à vérifier dans les laboratoires quelques-unes de ses vues les plus ingénieuses; les sociologues de la nouvelle manière discutent toujours ses idées avant de les écarter, cependant que les économistes réputés s'inspirent plus ou moins explicitement des faits qu'il a recueillis; surtout sa biologie est encore prise très au sérieux par ceux qui la connaissent. Enfin sa doctrine du progrès est d'origine française : elle parle à notre imagination, à nos instincts humanitaires. Herbert Spencer nous apparaît à la fois dans une gloire de savant comme Darwin, dont nous le distinguons mal, et avec un prestige de philosophe comme Auguste Comte, dont nous le croyons 
volontiers un disciple. Son renom, en définitive, est surtout français. » Gaston Rageot, « Herbert Spencer et la philosophie de la vie ", Revue des Deux Mondes, n² 22, 1904, p. 808-843, p. 810.

24. Concernant ces contextes, voir aussi Dirk Hoeges, Literatur und Evolution: Studien zur französischen Literaturkritik im 19. Jahrhundert: Taine, Brunetière, Hennequin, Guyau, Heidelberg, Winter, 1980, qui tente une réhabilitation d'Hennequin face à « l'esthétique réductrice des causes sociologiques » (« soziologische[...] Ursachenästhetik », p. 120) d'Hippolyte Taine.

25. Voir Hennequin, La Critique scientifique, op. cit., spécialement p.110, et tout le paragraphe "Théorie de l'analyse sociologique de M. Taine », p. 93-123. En modifiant considérablement les théories spencériennes, Hennequin, qui loue les suppositions de la psychologie physiologique, évite de priver l'homme de quelques aspects qui sont plutôt chers à la psychologie spiritualiste : loi propre des institutions sociales vis-à-vis de la nature, indépendance de l'individu génial de l'influence de son environnement, bonne santé mentale du génie.

26. Herbert Spencer, Les Premiers principes, trad. par É. Cazelles d'après la $1^{\text {re }}$ édition anglaise de 1863, Paris, Germer Baillière, 1871, p. 384.

27. «Tous les historiens modernes ont remarqué cette progression de la liberté individuelle de penser, des temps anciens aux nôtres, et M. Herbert Spencer a nettement relevé ce fait.» Hennequin, La Critique scientifique, op. cit., p. 144 sq.

28. Hennequin, ibid., p. 192 sqq. Hennequin se réfère probablement à « La dialectique sociale ", article publié par Tarde dans la Revue philosophique, $n^{\circ} 26,1888$, p. 20-41 et p. 148-165. Les Lois de l'imitation ne paraissent qu'en 1890. Sur Hennequin et Tarde, voir aussi Antoine Compagnon : « La psychologie sociale de Tarde, partageant l'humanité en deux classes, les répétiteurs et les novateurs, permet, par l'inversion plutôt que de la dialectisation de la causalité tainienne, de réconcilier un déterminisme avec la foi dans les individus, les grands hommes qui font l'histoire, les génies de la littérature : cela est très explicite dans le commentaire qu'en fait Hennequin. » ( La Troisième République des lettres, de Flaubert à Proust, Paris, Seuil, 1983, p. 187.)

29. " C'est ici qu'il se sépare, après l'avoir jusqu'alors assez fidèlement suivi, de l'auteur de l' Histoire de la littérature anglaise, et qu'il discute le degré d'influence qu'exercent sur la production de l'œuvre d'art la "race" et le "milieu". [...] il a très bien montré que si quelques artistes ont subi l'influence du milieu dans lequel ils ont vécu, d'autres y ont échappé, ce qui équivaut à dire que cette influence, n'ayant rien de fixe et de constant, n'a rien non plus de vraiment scientifique. » Brunetière, « La Critique scientifique, par M. Émile Hennequin », op. cit., p. 220.

30. Brunetière, L'Évolution des genres, op. cit., p. 20.

31. Ibid., p. 22.

32. Ibid.

33. Brunetière, «La Critique scientifique, par M. Émile Hennequin », op. cit., p. 219.

34. Dans la "Préface de l'auteur", Spencer ébauche le sujet de cette partie de la manière suivante: «[...] je montre que cette croyance à un absolu qui dépasse, non seulement la connaissance humaine, mais la conception humaine, est la seule base sur laquelle puisse s'établir la réconciliation de la religion et de la science. " Spencer, Les Premiers principes, op. cit., p. CXICXII.

35. Pour la théorie de l'«Inconnaissable » de Spencer et son succès en France voir aussi Daniel Becquemont et Laurent Mucchielli, La Cas Spencer, op. cit., p. 112-119.

36. Ferdinand Brunetière, "La métaphysique positiviste ", Revue des Deux Mondes, $\mathrm{n}^{\circ}$ 11, 1902, p. 578-601, ici p. 596 ; la formule citée est souvent reprise dans d'autres textes de Brunetière.

37. Ferdinand Brunetière, «La moralité de la doctrine évolutive ", Revue des Deux Mondes, $\mathrm{n}^{\circ} 129$, 1895, p. 136-162, ici p. 161. 
INDEX

Mots-clés : Taine (Hippolyte), Hennequin (Émile), Brunetière (Ferdinand), critique scientifique

\section{AUTEUR}

MARIE GUTHMÜLLER 